See discussions, stats, and author profiles for this publication at: https://www.researchgate.net/publication/338069987

\title{
Nibble-Based Face Recognition Using Convolution of Hybrid Features
}

Conference Paper · July 2019

DOI: 10.1109/IIISPC.2019.8935846

CITATION

1

3 authors, including:

\section{Raja K B}

University Visvesvaraya College of Engineering

188 PUBLICATIONS 1,346 CITATIONS

SEE PROFILE

Some of the authors of this publication are also working on these related projects:

Project Face recognition View project

Project Discovery of loT Objects View project
READS

51

Venugopal KR

University Visvesvaraya College of Engineering

925 PUBLICATIONS 3,717 CITATIONS

SEE PROFILE 


\title{
Nibble-Based Face Recognition Using Convolution of Hybrid Features
}

\author{
Pattarakamon Rangsee \\ Dept. of ECE \\ University Visvesvaraya College of Engineering, Bangalore \\ University \\ Bangalore, India \\ e-mail: pattarakamon@gmail.com
}

\author{
K. B. Raja \\ Dept. of ECE \\ University Visvesvaraya College of Engineering, Bangalore \\ University \\ Bangalore, India \\ e-mail: raja_kb@yahoo.com
}

\author{
Venugopal K. R. \\ Vice-Chancellor \\ Bangalore University \\ Bangalore, India \\ e-mail: venugopalkr@gmail.com
}

\begin{abstract}
Face Recognition technique is used to identify human beings effectively without their intervention. In this paper, we propose a novel nibble-based face recognition using convolution of hybrid features. Face images are converted from color to grayscale anda decimal values of each pixel is represented using 8-bit binary. The novel technique of converting 8-bit binary pixel into Left-Side Nibble (LSN) and Right-Side Nibble (RSN) is introduced to increase computational speed. The 4-bit LSN is converted to a decimal value varying between 0 and 240 . DWT is applied on LSN matrix and only $L L$ band coefficients are considered as first set of transform domain features. The 4-bit RSN is considered and converted into decimal values ranging between 0 and 15 . HOG is applied on RSN matrix to generate second set of spatial domain features. The final hybrid features are generated by performing linear convolution on spatial- and transformdomain features. The Artificial Neural Network (ANN) is used to compare and classify the database image features and test image features to compute performance parameters of proposed algorithm. It is observed that the performance of the proposed method is better than the existing methods.
\end{abstract}

Keywords- Biometrics; face recognition; Nibble; DWT; HOG

\section{INTRODUCTION}

The Pose Illumination and Expression (PIE) variant face recognition is a challenging task for biometric security system, which has a lot of potential in different application domains including forensics, law enforcement, and entertainment. The individual's face is recognized using sufficient training samples with effective feature extraction techniques. However, in the real-time, the images of each person are disguised and corrupted due to many reasons. Hence, face recognition with PIE variant is focused in this paper.

The key steps involved in requirement of the robust face recognition system are feature extraction and classification algorithms. Broader classification of feature extraction techniques includes spatial domain, frequency domain and hybrid domain. In the spatial domain, the image pixels are directly manipulated to obtain spatial domain features. The examples of spatial domain feature extraction techniques are Histogram of Oriented Gradient (HOG) [1], Local Binary Pattern (LBP) [2], Local Ternary Pattern (LTP) [2] etc. The frequency domain techniques are based on converting spatial domain images into frequency domain images and frequency domain features are either obtained directly or by manipulating frequency coefficients. The examples of frequency domain feature extraction techniques are Discrete Wavelet Transform (DWT) [3], Discrete Cosine Transform [4] etc. In hybrid domain, the spatial domain and frequency domain techniques are combined to obtain final features which are more effective compared to individual features. Final features which are obtained from feature extraction are classified into one of several classes in classification steps.

In this paper, a nibble-based face recognition using convolution approach for the hybrid feature is proposed. The novel nibble technique is introduced to increase computational speed and also recognition rate. The database and test image pixel values are converted into binary and segmented into LSN and RSN. The DWT is applied on LSN and only LL band coefficients are considered for initial set of transform domain features, with compressed size. The HOG is applied on RSN to generate second set of spatial domain features based on edge information. Linear convolution is performed using two initial feature sets to generated final features.

This paper is organized as follows. Section II provides the literature survey of face recognition in PIE variant. The proposed method is given in Section III. Section IV provides the experimental results using ANN. Conclusion, discussion, and future work provided in Section V.

\section{LITERATURE SURVEY}

In this section, the literature survey on the feature extraction techniques in both spatial and frequency domains are described. Additionally, the classification techniques are also presented.

Huda Mady and Shadi M.S. Hilles [5] have proposed a face recognition based on video broadcast under PIE 
variations. Viola-Jones algorithm was used to enhance face authentication in an uncontrolled environment in the realtime system with good accuracy. The HOG and LBP techniques are combined to extract face features. The random forest classifier is used as a face image classifier. Rajput et al., [6] proposed an efficient algorithm for the recognition of face-photo images using matching sketches. The feature extraction technique based on HOG feature descriptor is applied on face-photos, based on a query sketch drawn by an artist. KNN classifier is used for matching photo face images. Melkye Wereta Tsigie et al., [7] have proposed a robust face recognition technique using LBP and HOG feature extractor and descriptor. The HOG and LBP methods are used to extract edge and texture features. The multiclass Support Vector Machine (SVM) is used to classify final features for robust face recognition. Hua Wang et al., [8] have proposed a face recognition system based on Local Difference Binary (LDB) and HOG. The LDB and HOG techniques are used to extract the local pattern features and edge features of face images, respectively. The SVM is used as a classifier.

Kaushik Sett et al., [9] have proposed a face recognition scheme based on spatial and temporal features. The spatial domain and the temporal features are obtained using Principal Component Analysis (PCA) and DWT. The KNN classifier is used as a classification. Govardhan Mattela and Sandeep K. Gupta [10] have proposed a feature extraction technique for automatic facial expression recognition from video image sequence under the varying illumination using Gabor-Mean-DWT. The unique edge and texture pattern of face are extracted by Gabor filter. Subsequently, dimension and redundancy are reduced using Mean-DWT feature reduction technique to increase the recognition rate. Rabul Saikia and Aditya Bihar Kandali [11] have proposed a face recognition using Elliptical Local Binary Pattern and DWT for different illumination conditions and facial expressions on frontal and non-frontal face images. The KNN classifier is used to classify and evaluate performance results. Zied Bannour Lahaw et al., [12] have proposed an image compression using 2D-DWT in a preprocessing stage. The input image for feature extraction is the LL subband of 2DDWT, which has significant information of original image. The extracted features are obtained using PCA, Linear Discriminant Analysis and Independent Component Analysis techniques and tested on ORL database. The SVM is used as a classification. Akram Alsubari et al., [13] have proposed an Indian face recognition with different facial expressions. The final features are obtained using DWT and LBP. The SVM is used for classifier.

\section{PROPOSED METHOD}

The proposed approach mainly includes pre-processing, bit endianness, feature extraction, and classification. The block diagram of proposed approach for face recognition is shown in Fig. 1.

\section{A. Pre-Processing}

The images are collected from face databases and RGB color images are converted into grayscale images with the pixel values ranging between 0 to 255 . The gray scales images with different sizes are resized to 200X180. The histogram equalization is performed on Extended YALE-B face images to adjust contrast and illumination.

\section{B. Bit Endianness}

The decimal values of each pixel are converted into binary of eight bits. The eight-bit binary of every pixel is divided into four bit named as Least Significant Bits (LSB) and four bit named as Most Significant Bits (MSB). The four LSB bits are considered as Right Side Nibble (RSN) and four MSB bits are considered as Left Side Nibble (LSN). The decimal equivalent values of four-bit RSN varies between 0 and 15 using (1). The decimal values which are equivalent to four RSN, insignificantly compared to original decimal values of an image. The decimal equivalent values of four-bit LSN varies between 0 and 240 using (2). The decimal values which are equivalent to four LSN, significantly compared to original values of an image.

$$
\begin{gathered}
\text { RSN Decimal }=\sum_{n=1}^{4} x(n)\left(2^{n-1}\right) \\
L S N \text { Decimal }=\sum_{n=1}^{4} x(n)\left(16 \times 2^{n-1}\right)
\end{gathered}
$$

where $\mathrm{n}$ is bit position.

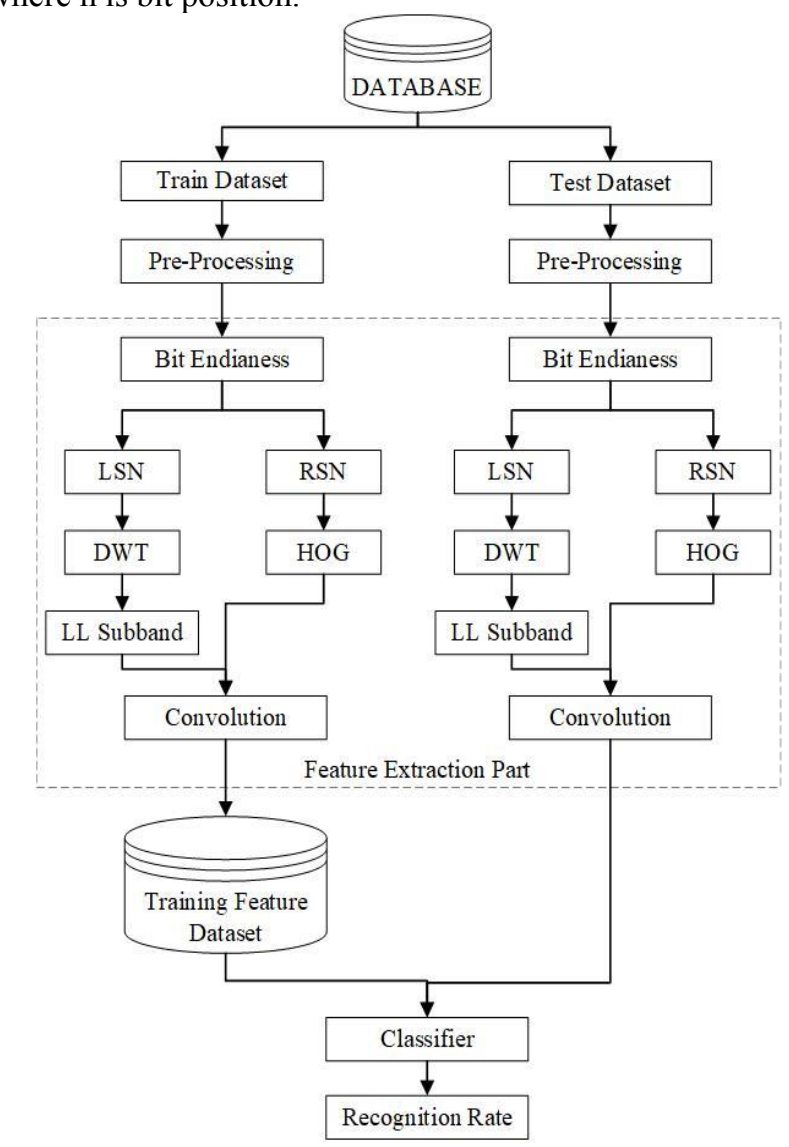

Figure 1. Block diagram of the proposed approach for face recognition

The advantage of bit endianness is converting the eightbit pixels into four-bit pixels. The features are extracted from 
four-bit pixels instead of eight-bit pixels, which increases the speed of computation and also decreases complexity real time system architecture.

\section{Feature Extraction}

1) Discrete Wavelet Transforms (DWT): In digital image processing, DWT is employed on rows of image using low pass filter and high pass filter concurrently. The detailed part (high frequency) and approximation part (low frequency) are obtained through filters and are down sampled by a factor of 2 . The operation is repeated on the columns of an image. The four sub-images obtained as approximation image (LL), vertical (LH), horizontal (HL) and diagonal $(\mathrm{HH})$ for every level of decomposition. The 2D-DWT is applied on LSN matrix to decompose it into four sub bands. The LL band image of LSN image is almost same as that of LSN image and consists of significant information. The horizontal, vertical and diagonal bands consist of horizontal, vertical and diagonal edge information which are insignificant information. The LL band coefficients are considered as initial features, since it has significant information of original image, and discarding three bands information. By considering only LL band features, the DWT technique rejects $3 / 4$ th coefficients i.e., LH, HL and HH, which results in better computational speed due to compression.

2) Histogram of Oriented Gradients (HOG): The HOG is applied on RSN matrix from bit endianness to extract features. First the horizontal and vertical gradients are filtered using kernels, $g_{H}$ and $g_{V}$, respectively. Then, it is used to obtain horizonal and vertical gradients to calculate the histogram of gradients. The gradients include magnitude (amplitude) and direction (angle) of each pixel values calculated using (3) and (4), respectively. The magnitude of gradient is high wherever there is a sharp change in intensity of the neighboring pixels.

$$
\begin{array}{r}
\text { Magnitude }=\sqrt{g_{H}^{2}+g_{V}^{2}} \\
\text { Direction }(\text { angle })=\arctan \left(\frac{g_{V}}{g_{H}}\right)
\end{array}
$$

where $g_{H}$ is horizontal gradient and $g_{V}$ is vertical gradient.

The RSN matrix is segmented into several blocks, where each block contains four cells and each cell contains $8 \mathrm{X} 8$ pixels. The histogram of gradients in each cell is computed and distributed into 9 bins of histogram which start from angle 0 to 180 degrees with unsigned gradients techniques. The range of each bin is 20 degrees. In each pixel, a bin is selected based on direction which is obtained from (4) then the values that goes into the bin is magnitude which is obtained from (3). The magnitudes are allotted to bins of histogram of gradients based on directions.

The RSN matrix of size $200 \mathrm{X} 180$ is segmented into $8 \mathrm{X} 8$ cells then HOG is applied on each cell to obtain $9 \mathrm{HOG}$ coefficients. The four adjacent cells of size $8 \mathrm{X} 8$ are grouped into one block of size $16 \mathrm{X} 16$. Each block has $9 \mathrm{X} 4=36$ HOG coefficients. The final features of RSN matrix are obtained by overlapping $50 \%$ of each block. The total number of overlapped blocks are $24 \mathrm{X} 21$ in RSN matrix. The total number of features are $24 \mathrm{X} 21 \mathrm{X} 36=18144$.

The RSN image consists of only four Least Significant Bits (LSB) hence it does not have significant information. The HOG visualization of RSN image has a random pattern without any significant information.

3) Fusion of initial features: The initial feature sets obtained from DWT and HOG are fused using linear convolution using (5) to derive final effective features for best face recognition. The DWT feature matrix of size $100 \times 90$ is converted into column vector of size $9000 \times 1$ and is represented using $\mathrm{D}(\mathrm{n})$, where $\mathrm{n}=0$ to 8999 . The HOG feature vector is of size 18144 and is represented using H(n), where $\mathrm{n}=0$ to 18143 . The final features are obtained using (5).

The total length of final features is equivalent to the length of DWT + length of HOG -1 i.e., total number of final features are 27143 .

$$
\begin{aligned}
& C(n)=D(n) * H(n) \\
& C(n)=\sum_{k=0}^{27142} D(k) H(n-k)
\end{aligned}
$$

\section{Aritificial Neural Network (ANN)}

In this paper, ANN is selected to classify the face images using proposed algorithm. ANNs are computational models capable of machine learning as well as pattern recognition. It has 3 layers i.e., input layer, hidden layer and output layer.

\section{EXPERIMENTAL RESULT ANALYSIS}

In this section, the definitions of performance parameters and the performance evaluation of the proposed method using ORL and Extended YALE-B face databases are discussed.

\section{A. Definitions of Performance Parameters for ANN}

The efficiency of the proposed model is shown by plotting the confusion matrix, which is the simplest metric used to determine the precision and accuracy of the model as shown in Fig. 2. There are four terms associated with confusion matrix to compare the cases between the actual class and the predicted class. They are True Positive (TP), True Negative (TN), False Positive (FP) and False Negative (FN).

\begin{tabular}{|c|c|c|c|}
\hline \multicolumn{2}{|c|}{ Confusion Matrix } & \multicolumn{2}{|c|}{ Actual (Model) } \\
\cline { 3 - 4 } & Positive & Negative \\
\hline \multirow{2}{*}{ Predict } & Positive & TP & FP \\
\cline { 2 - 4 } & Negative & FN & TN \\
\hline
\end{tabular}

Figure 2. Confusion matrix

1) Classification Accuracy: It is the proportion of the complete number of correct predictions against number of training images, evaluated using (6). 
Overall Accuracy $=\frac{T P+T N}{T P+F P+T N+F N}=\frac{\text { No.of Correct Match }}{\text { No. of Training Images }}$

2) Test Accuracy: It is the proportion of the complete number of correct predictions against number of testing images, evaluated using (7).

$$
\text { Test Accuracy }=\frac{\text { No.of Correct Match }}{\text { No. of Testing Images }}
$$

\section{B. The Experimental Results of Proposed Approach using ORL Database}

The Olivetti Research Laboratory (ORL) dataset is widely used in face recognition system with research. A total of 400 images were captured for forty subjects, ten images per subject and all the images are varied only in pose and facial expressions. Each image has a size of 112 X92 pixels in grayscale image.

The experimental results based on ORL dataset using proposed approach with ANN classifier is given in Table I. Firstly, the number of hidden nodes is varied by keeping Train Images Per Person (TIPP) constant. This helps to choose appropriate hidden nodes to find the efficiency of the proposed approach and it is observed that, 20 hidden nodes which result in a better overall accuracy as shown in first part of Table I. In the Second part, the best hidden nodes from part one are chosen and TIPP was varied to check the overall accuracy and error. It is observed that, by increasing TIPP, the percentage accuracy also varies.

TABLE I. VARIATIONS OF ACCURACY WITH A NUMBER OF HidDEN NODES AND TIPP USING AN ORL FACE DATASET

\begin{tabular}{ccc|cc}
\hline Control & $\begin{array}{c}\text { Hidden } \\
\text { Node }\end{array}$ & $\begin{array}{c}\text { No. of } \\
\text { TIPP }\end{array}$ & $\begin{array}{c}\text { Overall Acc. } \\
\text { (\%) }\end{array}$ & $\begin{array}{c}\text { Test Acc. } \\
(\%)\end{array}$ \\
\hline & 15 & 5 & 89.00 & 95.9163 \\
Constant & 20 & 5 & 90.00 & 96.9823 \\
TIPP & 22 & 5 & 87.50 & 96.2459 \\
& 25 & 5 & 89.25 & 96.6189 \\
& 27 & 5 & 85.75 & 95.9439 \\
Constant & 20 & 2 & 62.75 & 91.5694 \\
Hidden & 20 & 4 & 82.00 & 94.7045 \\
node & 20 & 5 & 90.00 & 96.9823 \\
& 20 & 6 & 95.00 & 97.8328 \\
& & & 98.00 & 99.2221 \\
\hline
\end{tabular}

C. The Experimental Results of Proposed Approach using Extended YALE B Database

The Extended Yale face database B (EYB) was released in 1984 and 2001 and images were captured from thirty-one subjects, with sixty-four images per subject and all the images are mainly varied illumination. Each image was cropped with a size of 192 X168 pixels in grayscale image.

The experimental results based on EYB dataset using proposed approach is shown in Table II. Firstly, the number of hidden nodes is varied by keeping TIPP constant. This helps to choose appropriate hidden nodes to find the efficiency of the proposed approach and it is observed that,
22 and 27 hidden nodes which have a better overall accuracy result as shown in first part of the Table II. In the Second part, hidden nodes of 22 from part one was chosen instead of 27, as less hidden nodes give a less computational time. With the constant 22 hidden nodes, TIPP was varied to check the overall accuracy and error. It is observed that by increasing TIPP, the percentage accuracy also increases.

TABLE II. VARIATIONS OF ACCURACY WITH A NUMBER OF HidDEN NODES AND TIPP USING AN EYB FACE DATASET

\begin{tabular}{ccc|cc}
\hline Control & $\begin{array}{c}\text { Hidden } \\
\text { Node }\end{array}$ & $\begin{array}{c}\text { No. of } \\
\text { TIPP }\end{array}$ & $\begin{array}{c}\text { Overall Acc. } \\
(\%)\end{array}$ & $\begin{array}{c}\text { Test Acc. } \\
(\%)\end{array}$ \\
\hline & 15 & 32 & 98.99 & 99.6657 \\
Constant TIPP & 20 & 32 & 98.99 & 99.6678 \\
& 22 & 32 & 99.40 & 99.8054 \\
& 25 & 32 & 99.04 & 99.7247 \\
& 27 & 32 & 99.40 & 99.8076 \\
Constant Hidden & 22 & 20 & 97.68 & 99.4871 \\
node & 22 & 32 & 99.40 & 99.8054 \\
& 22 & 40 & 99.65 & 99.9158 \\
& 22 & $\mathbf{5 0}$ & 99.85 & 99.9386 \\
\hline
\end{tabular}

D. Comparison of Proposed Algorithm with Existing Algorithms

The percentage accuracy of the proposed model is compared with the existing model and the corresponding values are given in Table III. It is shown that, the proposed model achieves significantly higher accuracy compared to the traditional methods. In order to justify the improvement in the performance parameter values of the proposed model, the following reasons have been stated:

- The number of gray shades for eight-bit binary are 256, which leads to architectural complexity and time-consuming process in real time applications. This disadvantage is eliminated by using LSN and RSN techniques in the proposed method and has only thirty-two (32) shades inplace of 256 shades.

- The histogram equalization to adjust the contrast and rid of illumination is applied to face images in the pre-processing stage in case of EYB dataset.

- DWT is applied on LSN, since it reduces the feature size to $1 / 4$

TABLE III. COMPARISON OF ACCURACY WITH PROPOSED AND EXISTING METHODS

\begin{tabular}{llc}
\hline Database & Method & Accuracy (\%) \\
\hline \multirow{2}{*}{ ORL } & Ying Wen [14] & 96.54 \\
& Abuzneid and Mahmood [15] & 96.90 \\
& Jun Fan et al., [16] & 97.10 \\
& Jun Kong et al. [17] & 97.50 \\
& Proposed Method & 98.00 \\
\hline \multirow{2}{*}{ EYB } & Santosh Kumar Jami et al. [18] & 96.70 \\
& Guangyi Chen et al. [19] & 97.30 \\
& Swarup Kumar Dandpat et al. & 97.64 \\
& [20] & 99.40 \\
& Weihong Deng et al. [21] & 99.85
\end{tabular}




\section{CONCLUSION}

Real-time face recognition system is required to identify human beings properly, with a smaller number of effective features. In this paper, a nibble-based face recognition based on convolution of hybrid features is proposed. The decimal values of each pixel in face images are converted into binary and further segmented into LSN and RSN. The LSN is converted back into decimal values which varies between 0 and 240, considering dominant information of an image. DWT is applied on LSN and only LL band coefficients are considered which has significant information of LSN matrix. Further a three-fourth compression of LSN matrix leads to a smaller number of effective feature coefficients. The RSN is converted into decimal values, varying between 0 to 15 , and HOG is applied on RSN matrix to generate second set of features with a smaller number of bits for each feature coefficients, which increases the speed of computation. The final features are computed by performing linear convolution on transform domain and spatial domain features. The final features of database and test section are classified using ANN, to compute performance parameters. It is observed that the proposed algorithm is better compared to the existing algorithms. The future work proposes to implement the proposed algorithm on real-time system considering 4 bits, in order to improve the computational time and reduce the complexity.

\section{REFERENCES}

[1] Aneta Kartali, Miloš Roglić, Marko Barjaktarović, Milica ĐurićJovičić and Milica M. Janković, "Real-time Algorithms for Facial Emotion Recognition: A Comparison of Different Approaches", 14th Symposium on Neural Networks and Applications (NEUREL), pp.1-4, 2018.

[2] Bin Xiao, Kaili Wang, Xiuli Bi, Weisheng Li and Junwei Han, "2DLBP: An Enhanced Local Binary Feature for Texture Image Classification", IEEE Transactions on Circuits and Systems for Video Technology, pp.1-1, 2018.

[3] Thamizharasi Ayyavoo and Jayasudha John Suseela, "Illumination pre-processing method for face recognition using 2D DWT and CLAHE”, IET Biometrics, Vol. 7, Issue 4, pp. 380-390, 2018.

[4] Taif Alobaidi and Wasfy B. Mikhael, "Face recognition system based on features extracted from two domains", IEEE 60th International Midwest Symposium on Circuits and Systems, pp. 977-980, 2017.

[5] Huda Mady and Shadi M. S. Hilles, "Face recognition and detection using Random forest and combination of LBP and HOG features", International Conference on Smart Computing and Electronic Enterprise (ICSCEE), pp. 1-7, 2018.

[6] G.G. Rajput, Prashantha and B. Geeta, "Face Photo Recognition from Sketch Images Using HOG descriptors", International Conference on Inventive Communication and Computational Technologies (ICICCT), pp. 555-558, 2018

[7] Melkye Wereta Tsigie, Rasika Thakare and Rahul Joshi, "Face Recognition Techniques Based on 2D Local Binary Pattern,
Histogram of Oriented Gradient and Multiclass Support Vector Machines for Secure Document Authentication", International Conference on Inventive Communication and Computational Technologies (ICICCT), pp. 1671-1676, 2018,

[8] Hua Wang, DingSheng Zhang and ZhongHua Miao, "Fusion of LDB and HOG for Face Recognition", Chinese Control Conference (CCC), pp. 9192-9196, 2018.

[9] Kaushik Sett, Pritam Das, Prosun Ghosh, Surya Kanta Ghosh, Aritra Dey, Neelanjan Saha and Suparna Biswas, "Face recognition using fusion of spatial and temporal features", Emerging Trends in Electronic Devices and Computational Techniques (EDCT), pp. 1-4, 2018.

[10] Govardhan Mattela and Sandeep K. Gupta, "Facial Expression Recognition Using Gabor-Mean-DWT Feature Extraction Technique", International Conference on Signal Processing and Integrated Networks, pp. 575-580, 2018.

[11] Rabul Saikia and Aditya Bihar Kandali, "DWT-ELBP based model for face recognition", International Conference on Energy, Communication, Data Analytics and Soft Computing, pp. 1348-1352, 2017

[12] Zied Bannour Lahaw, Dhekra Essaidani and Hassene Seddik, "Robust Face Recognition Approaches Using PCA, ICA, LDA Based on DWT, and SVM Algorithms", 41st International Conference on Telecommunications and Signal Processing (TSP), pp.1-5, 2018.

[13] Akram Alsubari, D. N. Satange and R. J. Ramteke, "Facial expression recognition using wavelet transform and local binary pattern", International Conference for Convergence in Technology (I2CT), pp. 338-342, 2017.

[14] Ying Wen, "A novel dictionary based SRC for face recognition", IEEE International Conference on Acoustics, Speech and Signal Processing, pp. 2582-2586, 2017

[15] Mohannad Abuzneid and Ausif Mahmood, "Face Recognition Framework Based on Correlated Images and Back-Propagation Neural Network", IEEE/ACS 15th International Conference on Computer Systems and Applications (AICCSA), pp. 1-7, 2018

[16] Jun Fan, Qiaolin Ye and Ning Ye, "Enhanced Adaptive Locality Preserving Projections for Face Recognition", IAPR Asian Conference on Pattern Recognition (ACPR), pp. 594-598, 2017

[17] Jun Kong, Min Chen, Min Jiang, Jinhua Sun and Jian Hou, "Face Recognition Based on CSGF(2D)2PCANet", IEEE Access, Vol.6, pp. 45153-45165, 2018

[18] Santosh Kumar Jami, Srinivasa Rao Chalamala and Krishna Rao Kakkirala, "Cross Local Gabor Binary Pattern Descriptor with Probabilistic Linear Discriminant Analysis for Pose-Invariant Face Recognition", UKSim-AMSS 19th International Conference on Computer Modelling \& Simulation (UKSim), pp. 39-44, 2017.

[19] Guangyi Chen, Tien D. Bui and Adam Krzyżak, "Filter-based face recognition under varying illumination", IET Biometrics, Vol.7, Issue.6, pp. 628-635, 2018.

[20] Swarup Kumar Dandpat, Sukadev Meher and Vivek Bopche, "Uneven Illumination Compensation for Unconstrained Face Recognition Using LBP”, International Conference for Convergence in Technology (I2CT), pp. 1-6, 2018

[21] Weihong Deng, Jiani Hu and Jun Guo, "Face Recognition via Collaborative Representation: Its Discriminant Nature and Superposed Representation", IEEE Transactions on Pattern Analysis and Machine Intelligence, Vol. 40, Issue. 10, pp, 2513-2521, 2018 